\title{
Presencia y estereotipos de grupos étnicos en los anuncios de prensa en Panamá Años 1998, 2008 y 2018
}

\author{
Tiffany Wang ${ }^{1 *}$ \\ ${ }^{1}$ Egresada de la Licenciatura en Ciencias de la Comunicación Social, Universidad Santa María La \\ Antigua, USMA.
}

*Autor para correspondencia. E-mail: wangtiffany.tw@gmail.com

Recibido: 24 de septiembre de 2019

Aceptado: 13 de noviembre de 2019

\begin{abstract}
Resumen
La investigación se enfoca en la presencia y los estereotipos que tienen los distintos grupos étnicos y cómo son proyectados en la publicidad panameña. El objetivo principal es evaluar la función que se le asigna a estos grupos en la publicidad impresa en los periódicos panameños y si son representados en roles que reiteran en los estereotipos. Se hizo una integración de métodos cuantitativos y cualitativos. Se realizó un análisis de contenido contabilizando la presencia de los grupos étnicos en la publicidad impresa en 360 ejemplares de los cuatro periódicos más populares de Panamá en tres décadas. Posteriormente, se entrevistaron seis directores creativos, quienes nos amplían el tema del uso de diversidad étnica en la publicidad en Panamá. La principal conclusión es que el grupo étnico que predomina en los avisos de prensa de Panamá son los blancos con 60.8\%, mientras que los mestizos tienen un cuarto de representación. Los afrodescendientes están presentes en $9.9 \%$ de la publicidad. Los grupos menos representados son los indígenas y los asiáticos con $1.4 \%$ y $1.1 \%$ de presencia respectivamente. Estas cifras en la publicidad no reflejan la realidad de distribución demográfica panameña. También se demostró que la participación de los grupos étnicos en la publicidad de los anuncios de prensa en Panamá, sí depende de la categoría y el nivel de prestigio del producto o servicio ofrecido por el anunciante.
\end{abstract}

Palabras clave: Publicidad, grupos étnicos, periódicos panameños, anuncios, estereotipos

\begin{abstract}
The research focuses on the presence and stereotypes that different ethnic groups have and how they are projected in Panamanian advertisement / media. The main objective is to evaluate the role assigned to these groups within the printed publicity in Panamanian newspapers and if they are represented in roles that relapse into stereotypes. An integration of quantitative and qualitative methods was made in which a content analysis was carried out. This entails 360 copies of the 4 most popular newspapers in Panama during a period of 3 decades. Subsequently, 6 creative directors were interviewed who broaden the topic of the use of ethnic diversity in Panama's advertisements. The main conclusion tells us that
\end{abstract}


the ethnic group that predominates Panama's press are the whites with $60.8 \%$, while the mestizos have a quarter of representation. Afro-descendants are present in $9.9 \%$. Whereas, the Indigenous group and Asians have a much inferior presence with $1.4 \%$ and $1.1 \%$ of participation respectively. In addition, these numbers in advertising is not a reflection of the reality of the Panamanian demographic distribution. Moreover, it was also shown that the participation of ethnic groups in the publicity of press advertisements in Panama, does in fact depend on the category and level of prestige of the product or service offered by the advertiser.

Keywords: advertisement, ethnic groups, Panamanian newspaper, stereotypes

\section{Introducción}

Panamá, siendo un país multicultural, ¿refleja la diversidad étnica en la publicidad dentro de la prensa escrita? Según los resultados del XI Censo Nacional de Población y VII de Vivienda realizado en el 2010 realizado por el Instituto de Estadística y Censo en el 2010, la población panameña está conformada por un $78.5 \%$ de mestizos, blancos y asiáticos, $12.3 \%$ de indígenas y $9.2 \%$ de afrodescendientes.

La publicación del periódico La Estrella de Panamá titulado "La pureza de nuestra diversidad" del 2015 detalla que el 68\% de la sociedad panameña es mestiza, El resto de la población está conformada por un $15 \%$ de blancos, $10 \%$ negros, $6 \%$ de ocho diferentes grupos indígenas y $1 \%$ de asiáticos, principalmente, de ascendencia china.

Sin embargo, podemos observar en la gran mayoría de publicidad impresa en los periódicos una poca participación de los distintos grupos étnicos y esto causa inequidad en la proporción de las etnias en los medios.

Podemos, en ocasiones, ver a los otros grupos étnicos, pero en un plano secundario y casi invisible. En los anuncios en donde están presentes, muchos son solo en contextos específicos retratando situaciones que inciden en los estereotipos tanto positivos como negativos que la sociedad les ha concedido a estos colectivos.

La investigación se centra en el eje de la representación de la diversidad étnica cultural incluyendo a los afrodescendientes, mestizos, blancos, indígenas y asiáticos utilizando la publicidad impresa en los periódicos como herramienta de medición. Identificar en los medios escritos qué tan seguido están presentes los diferentes grupos étnicos de Panamá en la publicidad en la prensa escrita y en qué contexto. Observar qué funciones interpretan y si sus roles son estereotipados.

La Ley 4 de 1999 sobre la Igualdad de Oportunidades para las Mujeres define los estereotipos como ideas, prejuicios, creencias y opiniones preconcebidos e impuestos por el medio social y cultura, que se aplica en forma general a todas las personas pertenecientes a la categoría a la que hacen referencia, como nacionalidad, etnia, edad o sexo.

Los estereotipos son una herramienta útil a la que acuden los publicistas y los ayuda en la comunicación usando significados simbólicos que se entienden fácilmente. Sin embargo, en muchas ocasiones el 
estereotipo se basa en una característica que es negativa o exagerada. Al hacerlo, esto disminuye al grupo a una "caricatura” (Wells, Moriarty \& Burnett, 2007, p. 76).

La publicidad no impone determinados valores ni los genera. En cambio, refleja los que predominan en la sociedad en la que se integra. Para ellos, no es posible que los anuncios traten de cambiar las creencias y los ideales de los consumidores, ya que uno de sus deberes es tratar de agradarles. Más bien, la publicidad refuerza los valores que ya están presentes en la vida social (Ortega et al., 2011)

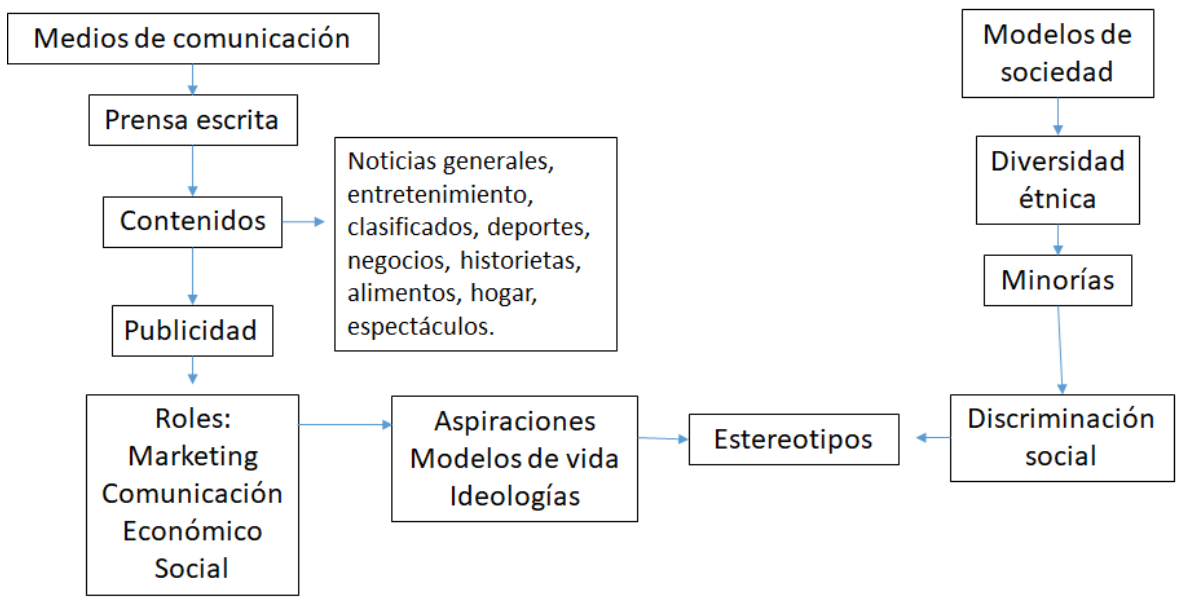

Figura 1. Esquema de elaboración propia con base en el planteamiento del problema

La prensa escrita es uno de los medios masivos más populares de comunicación. Desde su inicio, los periódicos ofrecen una amplia variedad de contenidos en donde se pueden encontrar las noticias generales, entretenimiento, clasificados, deportes, negocios, historietas, alimentos, hogar, espectáculos (Wells, Moriarty y Burnett, 2007). Para sustentar los costos de producción, recurren a la publicidad como su principal fuente de ingreso. La publicidad puede estar impresa en las páginas del periódico o insertadas en de este medio.

La función principal de "la publicidad consiste en informar a una o varias personas sobre un producto o servicio por medio de un anuncio pagado, con la intención de conseguir un objetivo" (Erickson, 2001, p.14). Pero según García y Thomas (2011) "la publicidad no solo vende productos, vende cultura e ideología" (p.115).

De acuerdo con Wells, Moriarty y Burnett (2007), la publicidad se compone de roles de marketing, de comunicación, económico y social. Enfocándonos en el rol de comunicación, los autores argumentan que es "una forma de comunicación masiva". Tiene el objetivo de conectar a los compradores y vendedores e informa sobre el producto o servicio. Viéndolo de la perspectiva de rol social, la publicidad "ayuda a formar una imagen de cada individuo al establecer modelos a seguir con los que cada quien se puede identificar".

Codeluppi (2007) resalta que la publicidad además "desempeña una gran importancia en el plano social. Incluso, podría decirse que es uno de los actores más potentes que actúan en el proceso de la producción de la cultura de la sociedad contemporánea" (p. 149-155). 
Díez Aguado (2015) afirma que la publicidad es como "un proceso de representación, capaz de generar imágenes, narrativas y discursos de la sociedad en la que vivimos" (p.11).

Dentro de los modelos de la sociedad, existe la diversidad étnica-cultural. Algunos grupos étnicos son más numerosos que otros. Las agrupaciones con menos personas se convierten en minorías con poco poder. Los que poseen ese dominio, Van Dijk (2001) los clasifica como élites, quienes son los que "controlan las dimensiones y decisiones más cruciales de la vida cotidiana de las minorías de los inmigrantes" (p.191).

Al ser un grupo minoritario, sufren discriminación social y son víctimas de estereotipos. Los medios de comunicación ocasionalmente refuerzan estos estereotipos encajonando a la sociedad a tener percepciones erróneas y generalizadas a la vez favoreciendo los patrones discriminatorios contra las minorías étnicas. (Jaramillo, Castillo, \& García, 2012).

En el 2016, se publicó el VI Informe Clara González (2011-2013) que describe que, a través de la publicidad, la imagen de la mujer es estereotipada en los roles tradicionales que la sociedad le ha asignado. Según el informe, Panamá sigue manejando un discurso racista y dominante en su publicidad y en los medios de comunicación; no se refleja la diversidad cultural, de género y étnica.

La publicidad tiene la capacidad de formar nuestros valores y nuestra opinión del mundo. Es por esta razón que los anunciantes y agencias de publicidad estén anuentes de cómo presentan los diferentes grupos étnicos en sus pautas.

Recíprocamente, si creemos que la publicidad refleja la sociedad, también está bajo la custodia de los anunciantes de asegurarse de que lo que presentan en los medios masivos sea preciso y representativo.

\section{Materiales y métodos}

Se estará incorporando la combinación de métodos de investigación. Tratamos de integrar subsidiariamente el método del análisis de contenido con la entrevista. El objetivo de utilizar la estrategia de combinación, es reforzar la validez de ambos métodos, compensando sus propias debilidades incorporando informaciones que proceden de la aplicación del otro método, aumentando las fortalezas de la investigación (Bericat, 1998).

La investigación tendrá un enfoque cuantitativo y cualitativo, utilizando el análisis de contenido como método de recolección de los datos. Para el desarrollo de la primera parte de la investigación, se trabajará con categorías de análisis para los periódicos. De acuerdo con Wells (2007), la publicidad dentro de los periódicos se clasifica en: clasificado, exhibición y suplementos. Nos enfocaremos en los de exhibición porque son los "predominantes" y en los que visualmente se puede analizar la presencia de los modelos. Estos se encuentran en cualquier parte del periódico y existen de todos los tamaños. Estaremos analizando todas las secciones de los diarios que contengan este tipo de publicidad.

Los periódicos son clasificados por el tamaño: el tabloide de 4 a 5 columnas por 32 centímetros y estándar, con 8 columnas por 54 centímetros (Treviño, 2010 \& Wells, 2007). Hemos escogido Crítica 
y El Siglo para el formato tabloide junto a La Prensa y La Estrella de Panamá, para el estándar, ya que son los que tienen mayor circulación en el país y contienen pautas durante las tres décadas que planeamos hacer la revisión.

Los periódicos a analizar serán de los años 1998, 2008 y 2018. Esto nos permite comparar y contrastar la publicidad a través de los años y observar si existen cambios en los conceptos creativos en los avisos de prensa. De la misma manera, nos permitirá revisar si con el tiempo se incrementa o disminuye la presencia de los numerosos grupos étnicos como modelos de estas campañas y cómo estos son representados, en qué circunstancias se encuentran y si reinciden en el uso de los estereotipos.

En la segunda parte, resaltando la parte cualitativa de la investigación, se harán entrevistas semiestructuradas a los expertos en las diferentes áreas de publicidad. Ellos tratarán de explicar los resultados encontrados con el análisis de la publicidad impresa en los periódicos porque son especialistas que están familiarizados en ese entorno. Se realizaron entrevistas a seis directores creativos quienes son los que proponen ideas y conceptos para las pautas, así como qué modelos utilizar. Este grupo de profesionales permitió sustentar las tendencias que se encontraron con el análisis de los periódicos y ampliar la investigación con sus perspectivas y experiencias.

Los encargados de esta función son:

- Roberto Varela: Vicepresidente Creativo en Unión DDB Panamá. Cuenta con 15 años como Director Creativo. Trabaja con marcas como Do It Center, Digicel, Conway, Bonlac, Caja de Ahorros y Super Xtra.

- Omar Polo: Vicepresidente Creativo de McCann Panamá. Con 12 años en publicidad, sus ideas han obtenido premios en festivales como Clio, D\&Ad, New York Shows, Facebook Awards, Fiap, El Sol, El Ojo de Iberoamérica y Effie, en otros

- Luciano Carrizo: Director Creativo en McCann Panamá, está encargado de todas las campañas de MasterCard en Centroamérica.

- Julio Flores: Director Creativo Digital de McCann Panamá. Crea contenido para marcas como Estrella Azul, Banisi y MasterCard Centroamérica.

- Sussie Salado: Directora Creativa en La Unión Publicitaria. Produce estrategias para clientes como Cervecería Nacional, Nestlé, Banco General, Grupo Melo.

- Jan Valdés: Director Creativo con 20 años de trayectoria. Actualmente labora en Trópico Ideas y Comunicación.

\subsection{Población y muestra}

Los diarios serán nuestra población. Cada año, se obtienen 365 publicaciones de La Prensa, La Estrella de Panamá, La Crítica y El Siglo. Es decir, en las tres décadas obtendremos 1,095 ejemplares por periódico dando un total de 4,380 que sería la población.

Para obtener la muestra, se utilizó el método del muestreo aleatorio simple con una población finita. Con un muestreo aleatorio simple, nos permite determinar el tamaño de una muestra con el fin de obtener una precisión dada (García Ferrando, 2000). 
Al saber que nuestra población es finita, la fórmula que se utiliza es:

En donde:

$$
n=\frac{z^{2} N p q}{e^{2}(N-1)+z^{2} p q}
$$

- $\mathrm{n}=$ tamaño de la muestra

- $\quad \mathrm{N}=$ tamaño de la población

- $\quad z=$ nivel de confianza a la cual asignamos para esta investigación el 95\%, siendo 1.96

- $\mathrm{p}=$ probabilidad que ocurra, en este caso es $5 \%=0.05$

- $\quad \mathrm{q}=$ probabilidad que no ocurra, es $\operatorname{decir}(1-\mathrm{p})$

- $\quad \mathrm{e}=$ error que es del $5 \%$

La fórmula quedaría representada de esta manera:

$$
\begin{gathered}
n=\frac{(1.96)^{2}(4,380)(0.50)(0.50)}{(0.05)^{2}(4,380-1)+(1.96)^{2}(0.50)(0.50)} \\
n=\frac{(3.8416)(4,380)(0.50)(0.50)}{(0.0025)(4,379)+(3.8416)(0.50)(0.50)} \\
n=\frac{4206.552}{10.9475+0.9604} \\
n=\frac{4206.552}{11.91} \\
n \approx 353 \\
n \approx 360
\end{gathered}
$$

Luego de haber realizado la ecuación, tuvimos un resultado de 353 ejemplares que será lo que conforma la muestra. Se ha redondeado a 360 para que la distribución sea más equitativa. Como consecuencia, se examinarán 30 ejemplares de cada periódico en cada década.

Para obtener los días de los diarios que serán revisados, se utilizó el programa Microsoft Excel, para generar 30 números aleatorios. Estas comprenden las 30 fechas de los periódicos durante los años 2018, 2008 y 1998.

\subsection{Hipótesis}

La hipótesis principal es que la publicidad les asigna roles a los distintos grupos étnicos basándose en estereotipos de la sociedad.

Otra hipótesis es que la participación de los grupos étnicos en la publicidad de los anuncios de prensa en Panamá depende de la categoría y nivel de prestigio del producto o servicio ofrecido por el anunciante. 


\subsection{Operacionalización de variables}

\begin{tabular}{|c|c|c|}
\hline Variable & Definición & Indicadores \\
\hline $\begin{array}{l}\text { Grupo } \\
\text { étnico }\end{array}$ & $\begin{array}{l}\text { Grupo de personas que } \\
\text { comparten una cultura similar, } \\
\text { idioma, religión, antepasados y } \\
\text { otras características que a } \\
\text { menudo pasan de una generación } \\
\text { a la siguiente. }\end{array}$ & $\begin{array}{l}\text { - } \text { Mestizos } \\
\text { - Afrodescendientes } \\
\text { - Indígenas } \\
\text { - Blancos } \\
\text { - Asiáticos }\end{array}$ \\
\hline Roles & $\begin{array}{l}\text { Función que proyecta los } \\
\text { modelos en la publicidad. } \\
\text { Contexto y prominencia en el } \\
\text { que aparece en el aviso. }\end{array}$ & $\begin{array}{l}\text { - Locación de la persona } \\
\text { (Principal, secundario, de } \\
\text { fondo) } \\
\text { - Contexto en la que se } \\
\text { encuentra } \\
\text { - Prestigio del anunciante } \\
\text { (Alto, medio, bajo) } \\
\text { - Género (Femenino, } \\
\text { masculino) }\end{array}$ \\
\hline $\begin{array}{l}\text { Producto o } \\
\text { servicio }\end{array}$ & $\begin{array}{l}\text { El producto o servicio } \\
\text { promocionado en los avisos de } \\
\text { prensa. }\end{array}$ & - Categoría del producto \\
\hline
\end{tabular}

\section{Resultados}

\subsection{Presencia de grupos étnicos en la publicidad panameña}

Los resultados demuestran que predomina la presencia las personas blancas con un $61.9 \%$ sobre los otros grupos en los avisos de prensa en todas las décadas examinandas. Más de la mitad de los modelos en las pautas analizadas son personas con características caucásicas.

El segundo grupo más popular es el mestizo que abarca alrededor del $25.7 \%$, seguido por los afrodescendientes quienes cuentan con un $9.9 \%$ de presencia en la publicidad impresa. Si lo comparamos con los datos del XI Censo Nacional de Población y Agencia Central de Inteligencia de Estados Unidos, los grupos étnicos presentados en la publicidad no tienen concordancia con la distribución de la población panameña. La participación de los indígenas y los asiáticos son casi invisibles abarcando solo el 1.4\% y 1.1\%, respectivamente. El análisis ha demostrado que la publicidad tiene una preferencia en utilizar los modelos de tez clara. 


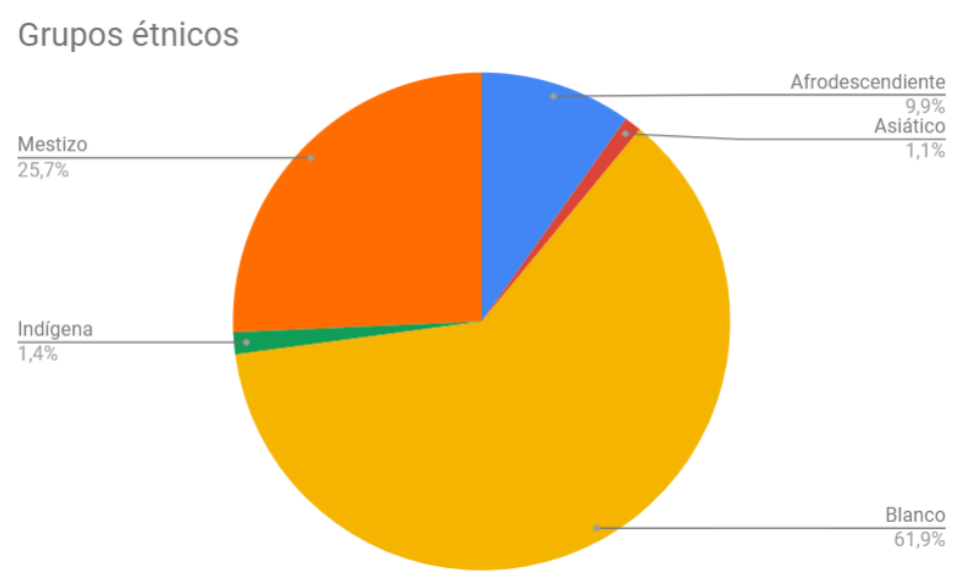

Figura 2. Presencia de grupos étnicos en los avisos de prensa de los años 1998, 2008 y 2018

Antes de mostrar los resultados, cuatro de seis creativos concordaron que la mayoría de las personas que aparecen en la publicidad son principalmente blancas.

"La mayoría de los clientes nos exigen usar gente blanca. En la mayoría de los casos que parezcan latinos. Como gente normal, panameña”. Roberto Varela

"En la publicidad panameña, creería que es el grupo étnico blanco" ... "La gente con rasgos más americanizados o europeizados". Omar Polo

"He visto más que todo blancos". Julio Flores

"En la publicidad panameña tradicionalmente se utiliza siempre o mayoritariamente modelos de tez blanca". Jan Valdés

Los otros dos describieron el uso de mestizos para reflejar la sociedad.

"Generalmente, más que nada son un mestizaje". Luciano Carrizo

"En este momento, yo creo que hay un esfuerzo que se está haciendo de las marcas por mostrar un grupo étnico más variado y que se vea más panameño, entre comillas, porque es muy difícil encasillar al panameño". Sussie Salado

Aun teniendo la mayor presencia en la publicidad de los periódicos examinados, el grupo de las personas blancas ha reducido su porcentaje de 62.3\% que tenía en 1998 a 56.2\% en el 2018. Esto representa una diferencia de $6.1 \%$.

\subsection{Cambios de participación durante las diferentes épocas}

A través de las décadas, vemos un aumento en la participación publicitaria para unos grupos étnicos y disminución en uno, mientras que otros se han mantenido estables. 
Para el grupo afrodescendiente hubo incremento de 3.9\% de presencia en la publicidad. Pasó de un $9.2 \%$ en el año 1998 a un $13.1 \%$ en el 2018. Entre las categorías de productos donde aparecen con más frecuencia son en servicios financieros, en productos inmobiliarios y en productos y servicios tecnológicos. El creativo de Trópico Ideas y Comunicación añade que el incremento no es significativo en términos de representación de la etnia negra.

"Quizás ha incrementado la presencia de las personas de raza negra, pero siento que no es representativa o no refleja la realidad de nuestra sociedad”. Jan Valdés

Los indígenas tuvieron un incremento en la representación en los avisos de prensa. Al analizar la muestra, se demostró un alza de 1.5\% de modelos mostrados en los periódicos en los últimos 20 años. En 1998, eran presentados en anuncios de oenegés, instituciones gubernamentales. En la década actual, su presencia se ha concentrado en los anuncios mágico -religioso.

Teniendo la mayor presencia en la publicidad de los periódicos examinados, el grupo de las personas blancas ha reducido su porcentaje de 62.3\% que tenía en 1998 a 56.2\% en el 2018 que representa diferencia de $6.1 \%$.

Aun siendo el grupo étnico que abarca más la población panameña, los mestizos solo tienen un cuarto de participación en la publicidad impresa de los periódicos. Los mestizos pasaron de tener una presencia de $26.5 \%$ hace 20 años a un $26.9 \%$ en 2018. Esto indica un leve incremento de $0.4 \%$.

El grupo de los asiáticos estuvo estable. Tuvo un incremento de $0.2 \%$ del 1998 al 2008. Sin embargo, disminuyó un $0.1 \%$ de en la última década.

Las entrevistas reflejan que los directores creativos proyectan un cambio en la inclusión y participación de los grupos étnicos en los avisos gráficos, pero será "un proceso muy lento". Poco a poco se han pautado nuevos conceptos e ideas que incluyan la utilización de otros grupos, disminuyendo así la dominación de los modelos blancos dentro de los periódicos panameños. Agregan los expertos que la integración de campañas con diversidad étnica será más frecuente y es una oportunidad para sus clientes.

\subsection{Roles de los grupos étnicos}

Tabla 1. Presencia de grupos étnicos en los avisos de prensa en los años 1998, 2008 y 2018

\begin{tabular}{|l|l|l|l|l|}
\hline Grupo étnico & Principal & Secundario & Fondo & Total \\
\hline & $47.2 \%$ & $6.5 \%$ & $8.2 \%$ & $61.9 \%$ \\
Blanco & $(1,351)$ & $(185)$ & $(235)$ & $(1,772)$ \\
\hline \multirow{3}{*}{ Mestizo } & $18.5 \%$ & $3.9 \%$ & $3.3 \%$ & $25.7 \%$ \\
\hline & $(529)$ & $(113)$ & $(93)$ & $(735)$ \\
\hline & $7.2 \%$ & $1.4 \%$ & $1.3 \%$ & $9.9 \%$ \\
Afrodescendiente & $(205)$ & $(39)$ & $(38)$ & $(285)$ \\
\hline & $1.2 \%$ & $0.1 \%$ & $0.2 \%$ & $1.4 \%$ \\
Indígena & $(34)$ & $(2)$ & $(5)$ & $(41)$ \\
\hline
\end{tabular}




\begin{tabular}{|l|l|l|l|l|}
\hline & $0.8 \%$ & $0.2 \%$ & $0.1 \%$ & $1.1 \%$ \\
Asiático & $(22)$ & $(6)$ & $(4)$ & $(32)$ \\
\hline & $74.8 \%$ & $12.1 \%$ & $13.1 \%$ & $100.0 \%$ \\
Total & $(2,141)$ & $(345)$ & $(375)$ & $(2,681)$ \\
\hline
\end{tabular}

Todos los grupos étnicos poseen roles protagónicos, secundarios y de fondo. La gran mayoría protagoniza las piezas publicitarias. Casi la mitad de los roles principales son personificados por las personas blancas con un $47.2 \%$. En segundo lugar, con un $18.5 \%$, tenemos a los mestizos en roles principales de los avisos de prensa y le siguen los afrodescendientes que tienen un $7.2 \%$ de modelos que aparecen en todas las piezas analizadas. De último, están los grupos indígenas y los asiáticos. En ese mismo orden, coinciden para los papeles secundarios y de fondo.

\subsection{Nivel de prestigio de los productos y servicios de los anunciantes}

\section{Alto}

Los blancos dominan la categoría de los productos y servicios de nivel de prestigio alto. Más de la mitad de los modelos que se encontraron de esta categoría poseen un papel principal con un $66.0 \%$. Encabezan la lista de los roles secundarios y de fondo con $8.4 \%$ y $6.4 \%$ respectivamente. "La publicidad históricamente fue aspiracional y dentro de esa aspiracionalidad escondió muchos estereotipos, muchos estigmas a determinadas razas y debido a un componente de querer ser como el hombre blanco", comentó Omar Polo mientras que Sussie Salado explica que "cuando vas a hablar bajo una marca que les habla a personas que son de mejor clase social, se busca gente más blanca".

El segundo grupo étnico más representado en esta categoría alta son los mestizos. En un rol principal, ellos tienen una presencia del 10.0\%. Los indígenas no aparecen en el nivel de prestigio alto en ninguna de las locaciones.

\section{Medio}

Para el nivel de prestigio medio, las personas blancas vuelven a encabezar en los roles principales, secundarios y de fondo. Casi la mitad se encuentra en el papel principal con un 47.4\%. Los mestizos los sigue en un $19.4 \%$ en donde ocupan un rol principal en la gama media de prestigio. Hay una participación mínima de parte de los indígenas y de los asiáticos en esta categoría.

\section{Baja}

Los mestizos son los más utilizados para representar a los productos y servicios de prestigio bajo. Un cuarto de los modelos de esta categoría son las personas mestizas, las que tienen el papel principal. Los blancos es el segundo grupo con un $22.8 \%$ en un rol principal. Le siguen los afrodescendientes, los indígenas y finalmente, está el grupo de los asiáticos, con poca presencia en este nivel de prestigio.

A través de los años la figura blanca era utilizada como la ideal para representar productos y servicios lujosos, de altos costos y aspiracionales. Sussie Salado detalla que los clientes buscan personas de tez más clara para proyectar una clase social alta. 
Tabla 2. Comparativa de presencia de los grupos étnicos dentro de cada categoría de producto

\begin{tabular}{|c|c|c|c|c|c|c|}
\hline \multirow[b]{2}{*}{$\begin{array}{l}\text { Categoría de } \\
\text { producto }\end{array}$} & \multicolumn{6}{|c|}{ Grupo étnico } \\
\hline & Afro & Asiático & Blanco & Indígena & Mestizo & Total \\
\hline $\begin{array}{l}\text { Servicios } \\
\text { financieros }\end{array}$ & $\begin{array}{l}9.4 \% \\
(53)\end{array}$ & $\begin{array}{l}0.7 \% \\
(4)\end{array}$ & $\begin{array}{l}65.5 \% \\
(369)\end{array}$ & $\begin{array}{l}0.2 \% \\
(1)\end{array}$ & $\begin{array}{l}24.2 \% \\
(136)\end{array}$ & $\begin{array}{l}100.0 \% \\
(563)\end{array}$ \\
\hline $\begin{array}{l}\text { Ropa, zapatos y } \\
\text { accesorios }\end{array}$ & $\begin{array}{l}7.5 \% \\
(29)\end{array}$ & $\begin{array}{l}1.3 \% \\
(5)\end{array}$ & $\begin{array}{l}65.3 \% \\
(254)\end{array}$ & & $\begin{array}{l}26.0 \% \\
(101)\end{array}$ & $\begin{array}{l}100.0 \% \\
(389)\end{array}$ \\
\hline $\begin{array}{ll}\text { Productos } & \text { y } \\
\text { servicios } & \\
\text { tecnológicos }\end{array}$ & $\begin{array}{l}10.3 \% \\
(39)\end{array}$ & $\begin{array}{l}1.6 \% \\
(6)\end{array}$ & $\begin{array}{l}65.4 \% \\
(248)\end{array}$ & & $\begin{array}{l}22.7 \% \\
(86)\end{array}$ & $\begin{array}{l}100.0 \% \\
(379)\end{array}$ \\
\hline $\begin{array}{l}\text { Productos } \\
\text { inmobiliarios }\end{array}$ & $\begin{array}{l}14.2 \% \\
(41)\end{array}$ & & $\begin{array}{l}71.9 \% \\
(207)\end{array}$ & & $\begin{array}{l}13.9 \% \\
(40)\end{array}$ & $\begin{array}{l}100.0 \% \\
(288)\end{array}$ \\
\hline $\begin{array}{l}\text { Artículos del } \\
\text { hogar }\end{array}$ & $\begin{array}{l}5.7 \% \\
(11)\end{array}$ & $\begin{array}{l}0.5 \% \\
(1)\end{array}$ & $\begin{array}{l}75.6 \% \\
(146)\end{array}$ & $\begin{array}{l}0.5 \% \\
(1)\end{array}$ & $\begin{array}{l}17.6 \% \\
(34)\end{array}$ & $\begin{array}{l}100.0 \% \\
(193)\end{array}$ \\
\hline Autos & $\begin{array}{l}5.5 \% \\
(10)\end{array}$ & $\begin{array}{l}3.8 \% \\
(7)\end{array}$ & $\begin{array}{l}72.7 \% \\
(133)\end{array}$ & & $\begin{array}{l}18.0 \% \\
(33)\end{array}$ & $\begin{array}{l}100.0 \% \\
(183)\end{array}$ \\
\hline $\begin{array}{l}\text { Alimentos } \quad y \\
\text { bebidas }\end{array}$ & $\begin{array}{l}12.2 \% \\
(18)\end{array}$ & $\begin{array}{l}1.4 \% \\
(2)\end{array}$ & $\begin{array}{l}53.4 \% \\
(79)\end{array}$ & & $\begin{array}{l}33.1 \% \\
(49)\end{array}$ & $\begin{array}{l}100.0 \% \\
(148)\end{array}$ \\
\hline $\begin{array}{l}\text { Institución } \\
\text { gubernamental }\end{array}$ & $\begin{array}{l}10.4 \% \\
(14)\end{array}$ & & $\begin{array}{l}29.1 \% \\
(39)\end{array}$ & $\begin{array}{l}5.2 \% \\
(7)\end{array}$ & $\begin{array}{l}55.2 \% \\
(74)\end{array}$ & $\begin{array}{l}100.0 \% \\
(134)\end{array}$ \\
\hline Otros & $\begin{array}{l}7.7 \% \\
(10)\end{array}$ & & $\begin{array}{l}43.1 \% \\
(56)\end{array}$ & $\begin{array}{l}1.5 \% \\
(2)\end{array}$ & $\begin{array}{l}47.7 \% \\
(62)\end{array}$ & $\begin{array}{l}100.0 \% \\
(130)\end{array}$ \\
\hline $\begin{array}{l}\text { Servicios } \\
\text { educativos }\end{array}$ & $\begin{array}{l}13.5 \% \\
(14)\end{array}$ & $\begin{array}{l}0.9 \% \\
(1)\end{array}$ & $\begin{array}{l}59.5 \% \\
(66)\end{array}$ & $\begin{array}{l}0.9 \% \\
(1)\end{array}$ & $\begin{array}{l}25.2 \% \\
(28)\end{array}$ & $\begin{array}{l}100.0 \% \\
(111)\end{array}$ \\
\hline $\mathrm{ONG}$ & $\begin{array}{l}12.7 \% \\
(13)\end{array}$ & $\begin{array}{l}2.9 \% \\
(1)\end{array}$ & $\begin{array}{l}28.4 \% \\
(29)\end{array}$ & $\begin{array}{l}15.7 \% \\
(16)\end{array}$ & $\begin{array}{l}40.2 \% \\
(41)\end{array}$ & $\begin{array}{l}100.0 \% \\
(102)\end{array}$ \\
\hline Sector servicio & $\begin{array}{l}10.2 \% \\
(6)\end{array}$ & $\begin{array}{l}1.7 \% \\
(3)\end{array}$ & $\begin{array}{l}57.6 \% \\
(34)\end{array}$ & $\begin{array}{l}1.7 \% \\
(1)\end{array}$ & $\begin{array}{l}28.8 \% \\
(17)\end{array}$ & $\begin{array}{l}100.0 \% \\
(59)\end{array}$ \\
\hline $\begin{array}{ll}\begin{array}{l}\text { Artículo } \\
\text { cuidado }\end{array} & \text { de } \\
\text { personal } & \text { y } \\
\text { belleza } & \end{array}$ & $\begin{array}{l}2.4 \% \\
(1)\end{array}$ & $\begin{array}{l}2.4 \% \\
(1)\end{array}$ & $\begin{array}{l}90.5 \% \\
(38)\end{array}$ & & $\begin{array}{l}4.8 \% \\
(2)\end{array}$ & $\begin{array}{l}100.0 \% \\
(42)\end{array}$ \\
\hline $\begin{array}{l}\text { Ofertas de } \\
\text { trabajo }\end{array}$ & $\begin{array}{l}11.5 \% \\
(3)\end{array}$ & & $\begin{array}{l}53.8 \% \\
(14)\end{array}$ & & $\begin{array}{l}34.6 \% \\
(9)\end{array}$ & $\begin{array}{l}100.0 \% \\
(26)\end{array}$ \\
\hline $\begin{array}{l}\text { Medios de } \\
\text { comunicación }\end{array}$ & $\begin{array}{l}23.1 \% \\
(6)\end{array}$ & $\begin{array}{l}3.8 \% \\
(1)\end{array}$ & $\begin{array}{l}57.7 \% \\
(15)\end{array}$ & & $\begin{array}{l}15.4 \% \\
(4)\end{array}$ & $\begin{array}{l}100.0 \% \\
(26)\end{array}$ \\
\hline $\begin{array}{l}\text { Servicios de } \\
\text { salud }\end{array}$ & $\begin{array}{l}5.9 \% \\
(1)\end{array}$ & & $\begin{array}{l}70.6 \% \\
(12)\end{array}$ & & $\begin{array}{l}23.5 \% \\
(4)\end{array}$ & $\begin{array}{l}100.0 \% \\
(17)\end{array}$ \\
\hline Aerolínea & $\begin{array}{l}13.3 \% \\
(2)\end{array}$ & & $\begin{array}{l}66.7 \% \\
(10)\end{array}$ & & $\begin{array}{l}20.0 \% \\
(3)\end{array}$ & $\begin{array}{l}100.0 \% \\
(15)\end{array}$ \\
\hline $\begin{array}{l}\text { Servicios } \\
\text { mágico } \\
\text { religioso }\end{array}$ & $\begin{array}{l}14.3 \% \\
(2)\end{array}$ & & & $\begin{array}{l}85.7 \% \\
(12)\end{array}$ & & $\begin{array}{l}100.0 \% \\
(14)\end{array}$ \\
\hline
\end{tabular}




\begin{tabular}{|c|c|c|c|c|}
\hline $\begin{array}{l}\text { Anuncios de } \\
\text { suplementos }\end{array}$ & & $\begin{array}{l}90.0 \% \\
(9)\end{array}$ & $\begin{array}{l}10.0 \% \\
\text { (1) }\end{array}$ & $\begin{array}{l}100.0 \% \\
(10)\end{array}$ \\
\hline Evento & $\begin{array}{l}40.0 \% \\
(4)\end{array}$ & $\begin{array}{l}30.0 \% \\
(3)\end{array}$ & $\begin{array}{l}30.0 \% \\
(3)\end{array}$ & $\begin{array}{l}100.0 \% \\
(10)\end{array}$ \\
\hline $\begin{array}{l}\text { Servicios de } \\
\text { envío }\end{array}$ & $\begin{array}{l}20.0 \% \\
(2)\end{array}$ & $\begin{array}{l}30.0 \% \\
(3)\end{array}$ & $\begin{array}{l}50.0 \% \\
(5)\end{array}$ & $\begin{array}{l}100.0 \% \\
(10)\end{array}$ \\
\hline Constructoras & $\begin{array}{l}40.0 \% \\
(2)\end{array}$ & & $\begin{array}{l}60.0 \% \\
(3)\end{array}$ & $\begin{array}{l}100.0 \% \\
(5)\end{array}$ \\
\hline $\begin{array}{l}\text { Espacio } \\
\text { recreativo }\end{array}$ & & $\begin{array}{l}100.0 \% \\
(4)\end{array}$ & & $\begin{array}{l}100.0 \% \\
(4)\end{array}$ \\
\hline $\begin{array}{l}\text { Centros } \\
\text { comerciales }\end{array}$ & & $\begin{array}{l}100.0 \% \\
(3)\end{array}$ & & $\begin{array}{l}100.0 \% \\
(3)\end{array}$ \\
\hline
\end{tabular}

En esta tabla se presentan los resultados de la participación de los grupos étnicos en cada categoría de productos o servicios ofrecidos en las pautas. Las categorías en las que están presentes todos los grupos étnicos son en servicios financieros, artículos del hogar, servicios educativos, ONG y sector servicio.

Lo primero que podemos observar es que las personas blancas tienen participación en todas las categorías, menos en constructoras y servicios mágico religioso. Este es el grupo étnico que tiene mayor presencia en casi la mayoría de las categorías. Este grupo predomina en la publicidad que promociona la apertura de espacios recreativos $(100 \%)$, centros comerciales $(100 \%)$, artículos de cuidado personal y belleza $(90.5 \%)$ y en anuncios de suplementos $(90 \%)$.

Los mestizos son más utilizados para representar comerciales de constructoras $(60.0 \%)$, instituciones gubernamentales $(55.2 \%)$ y servicios de envío $(50.0 \%)$.

Los mestizos cada vez se están abriendo paso a ser el común denominador de lo que es la imagen del panameño. Los entrevistados afirman que son los tipos de modelos que generalmente buscan las marcas para representar sus productos y servicios.

"Trata de siempre mostrarse gente un poco trigueña, pelo oscuro, ojos oscuros también. Y creo que se ha estado rompiendo esa manía que había de antes querer mostrar siempre cosas irreales. Gente rubia, ojos azules todo el tiempo por querer subirle el nivel a las marcas". Sussie Saldo 

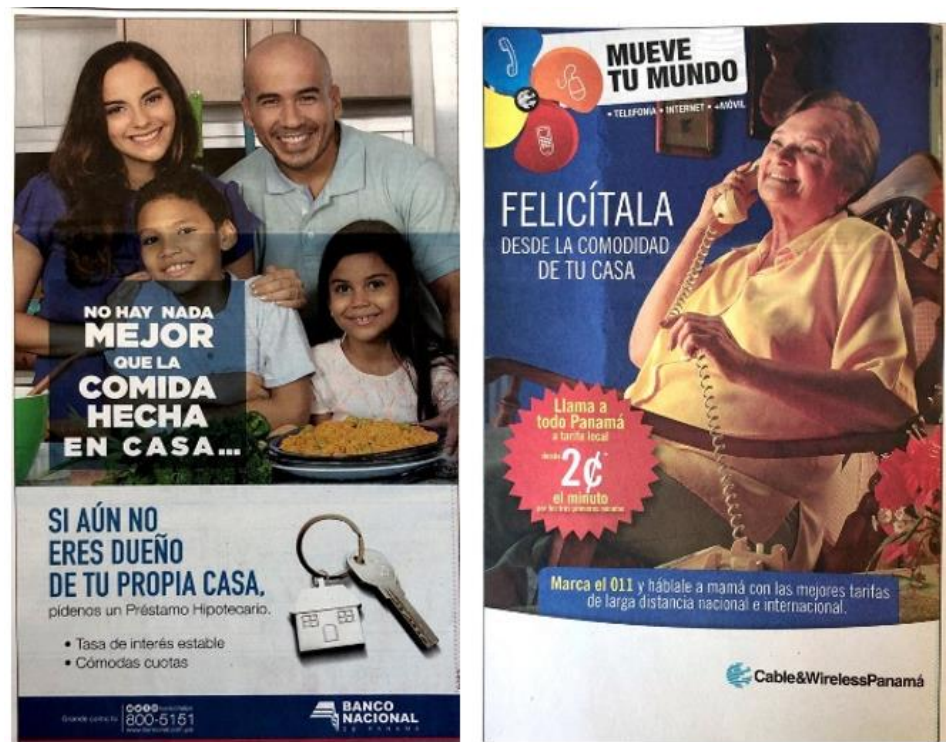

Figura 1. Ejemplo de presencia de mestizos en categoría de servicios financieros y de productos y servicios tecnológicos

El 40\% de los modelos en las pautas de eventos y constructoras son afrodescendientes. Otra área en la que podemos encontrar a este grupo es en la publicidad de medios de comunicación (23.1\%). Los afrodescendientes es el tercer grupo que tiene más presencia en la publicidad impresa en los últimos años, detrás de los blancos y mestizos. Se encuentran principalmente en roles relacionados con productos de prestigio medio.

El Director Creativo Omar Polo destaca que las personas afrodescendientes asumen papeles específicos dentro del mundo publicitario. "Al hombre negro se le adjudicaron determinadas características y estuvo en determinados roles en la publicidad y en determinadas marcas".

La categoría en donde dominan las personas indígenas es en la publicidad de servicios mágicoreligioso, con un $85.7 \%$. Este grupo solo aparece en 8 de 24 categorías de los anuncios analizados. Los anunciantes de ONG (15.7\%) e instituciones gubernamentales (5.2\%) son los que promueven la imagen de los grupos originarios de Panamá.

Los indígenas no aparecieron en el nivel de prestigio alto y este grupo tuvo una leve participación en el medio y bajo. Las categorías en donde se utilizaba la figura indígena son explícitas con el objetivo de mostrar precisamente, la diversidad cultural en Panamá. Jan Valdés agrega que dentro de la publicidad en general, la presencia de este grupo es nula. "Las personas indígenas casi que son invisibles en la publicidad a menos que se trate de algún tema específico, de turismo, de emprendimiento o de orgullo nacional". 

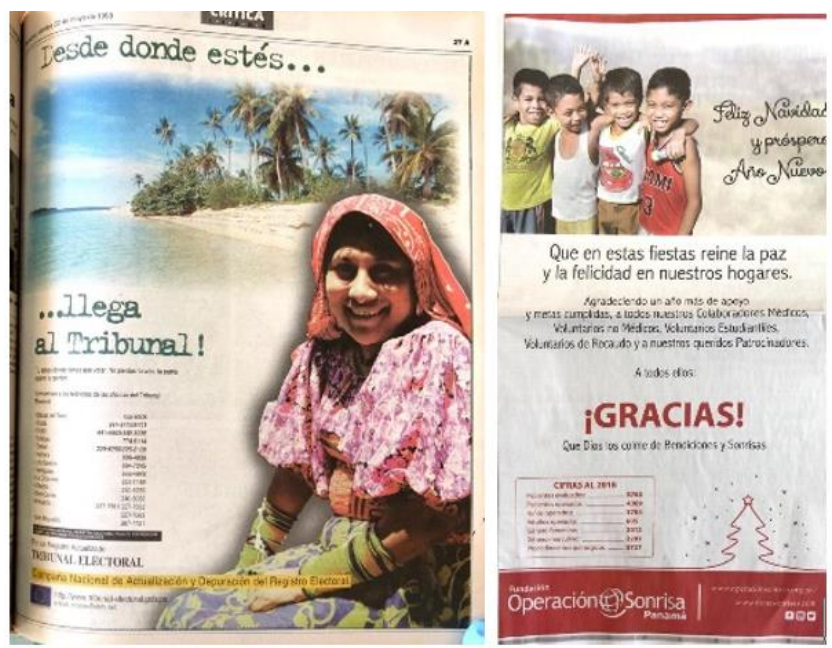

Figura 2. Ejemplo de presencia de indígenas en categoría de institución gubernamental y ONG

En el caso de los asiáticos, ellos tienen presencia en 10 de 24 categorías. El más alto es en la de los autos $(3.8 \%)$. Le sigue ONG $(2.9 \%)$ y los artículos de cuidado personal y belleza $(2.4 \%)$. Son los que menos participación tienen dentro de la publicidad panameña y rara vez se encontraban protagonizando roles principales sin estar acompañados de otros grupos étnicos.

Basándonos en los resultados obtenidos del análisis de contenido y las entrevistas, se pudo comprobar que la publicidad sí les asigna roles a los distintos grupos étnicos basándose en estereotipos de la sociedad.

Los seis directores creativos entrevistados coincidieron en que la publicidad en general trabaja con los estereotipos. Es un factor que ayuda a promocionar un producto o servicio, pero de la misma manera, refuerza esas generalizaciones.

"La publicidad alimenta los estereotipos y al mismo tiempo se alimenta de los estereotipos". Luciano Carrizo

"Es un camino de dos vías, pero, entre las dos, pienso que la publicidad es la que impone esos estereotipos". Jan Valdés

El contexto se refiere a en qué situación se encuentran las personas que aparecen en la publicidad. Las pautas sitúan a los grupos étnicos principalmente en escenarios de vida cotidiana y trabajo. Por otro lado, los avisos de prensa utilizan mucho la imagen de las personas en donde no aparecen con un fondo específico. A esos artes se le asignó la categoría de "sin fondo".

Los afrodescendientes, blancos y mestizos aparecen en todos los escenarios de contextos mientras que los asiáticos y los indígenas no están en situaciones de ocio, fiesta, ni escuela. Las marcas panameñas se inclinan por retratar la vida cotidiana de los individuos para promocionar sus productos y servicios. De acuerdo con la muestra, muy pocas veces vemos publicidad en los escenarios de fiestas o en escuelas. 
En los resultados obtenidos del análisis de contenido, se puede observar que es equitativa con una ligera diferencia de 1\% a favor de los hombres en cuestión de representación de género en la publicidad panameña. De la muestra, $49.5 \%$ son del sexo femenino, mientras que un $50.5 \%$, son del masculino.

Al comparar la representación de género en las tres décadas, podemos notar que la participación de las mujeres en la publicidad se ha ido incrementando. Hace 20 años, los hombres tenían mayor participación con una diferencia de $9.6 \%$ sobre las mujeres. Para el 2008 se invirtió y el sexo femenino supera la presencia del masculino por un $0.8 \%$. En la época actual, este número se incrementa a un $6.4 \%$.

Las cifras demuestran que la representación de las mujeres en la publicidad va en aumento. Las marcas analizadas cuyo grupo objetivo son mujeres están pautando más. Otro factor que se puede tomar en consideración del alza es que las mujeres, cada vez más, tienen poder económico.

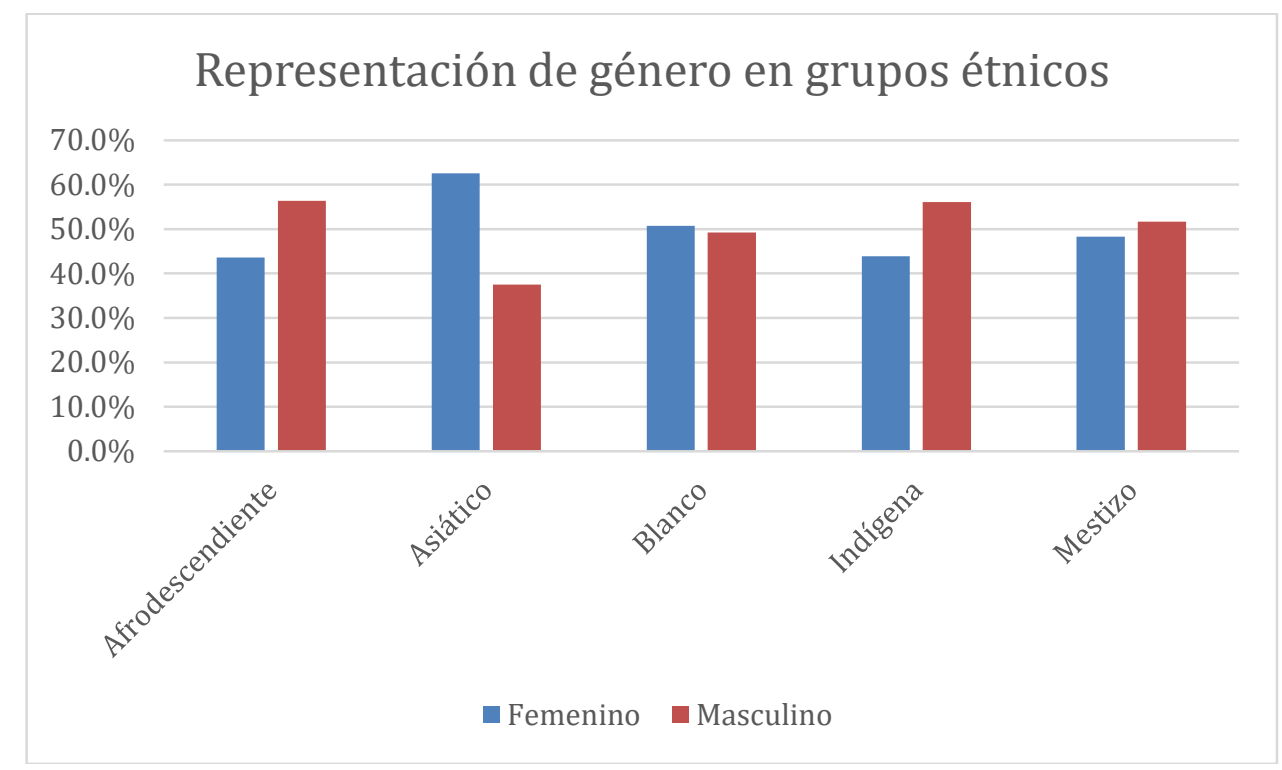

Figura 3. Comparación de géneros en los grupos étnicos

La participación de los hombres en los grupos de los afrdescendientes, indígenas y mestizos es superior a la de las mujeres, mientras que en los asiáticos y los blancos dominan las mujeres.

La diferencia más notable es en los asiáticos en los que las mujeres tienen mayor presencia por un 25\% sobre los hombres. Los hombres afrodescendientes e indígenas son más populares que sus contrapartes por un $12.8 \%$ y $12.2 \%$, respectivamente.

Los grupos con menos diferencias son los mestizos; los hombres son 3.4\% más utilizados que las mujeres y los blancos, grupo en el que el sexo femenino supera el masculino por un $1.6 \%$.

Se demuestra que los grupos étnicos más utilizados en la publicidad panameña poseen menos diferencia en la representación de género. En cambio, el auge es mayor en los demás grupos. 


\section{Conclusiones y recomendaciones}

La investigación demostró que la publicidad sí les asigna roles a los distintos grupos étnicos basándose en estereotipos de la sociedad. De acuerdo con las entrevistas, los seis directores creativos afirmaron que la publicidad sigue recurriendo a los estereotipos que ya existen en la sociedad y a la vez son reforzados por la pauta.

El grupo étnico que predomina en los avisos de prensa de Panamá son los blancos con $60.8 \%$ mientras que los mestizos tienen un cuarto de representación. Los afrodescendientes están presentes en 9.9\% de la publicidad. Los grupos menos utilizados son los indígenas y los asiáticos con 1.4\% y 1.1\% de presencia respectivamente. Estas cifras en la publicidad no son reflejo de la realidad de la distribución demográfica panameña.

La investigación también demostró otra hipótesis; la participación de los grupos étnicos dentro de la publicidad de los anuncios de prensa en Panamá sí depende de la categoría y el nivel de prestigio del producto o servicio ofrecido por el anunciante.

Los diversos grupos étnicos son integrados en la publicidad de la prensa escrita cuando las marcas tienen el objetivo de llegarles a esas personas en específico o cuando la marca tiene el fin de mostrarse como multicultural.

El estereotipo principal que se encontró es que las personas blancas reflejan belleza, prestigio y alto nivel adquisitivo. En el análisis de contenido se encontró que los blancos dominan en las pautas de nivel de prestigio alto y medio, mientras que los mestizos predominaban en los bajos. Los directores creativos concuerdan que la publicidad en el promociona campañas aspiracionales y que la figura del blanco es representación de esa aspiracionalidad.

La presencia de los grupos aborígenes de Panamá en la publicidad es mínima. No aparecen en marcas de prestigio alto y son protagonizados en pautas para servicios mágico religioso, ONG y de instituciones gubernamentales. Esto demuestra que los indígenas solo son tomados en cuenta para campañas que tiene como objetivo mostrar diversidad.

El grupo utilizado principalmente por las marcas para reflejar la imagen de panameños, son los mestizos.

El análisis de contenido de la publicidad muestra que en los últimos 20 años se ha registrado un leve incremento en la diversidad étnica en de la publicidad aumentando el número de personas mestizas, afrodescendientes, indígenas y asiáticos. Los directores creativos afirman que es un crecimiento lento.

En la categoría de servicios financieros la presencia de los blancos disminuye mientras que los afrodescendientes y mestizos aumentan. Lo mismo ocurre en el mercado de ropa, zapatos y accesorios. Se registra una reducción de $28.4 \%$ en los blancos y un alza de $21.6 \%$ para los mestizos. Sucede lo contrario en el área de productos inmobiliarios; el blanco sube el porcentaje y otros bajan.

Existe una tendencia de utilizar más a los mestizos para las pautas relacionadas con instituciones gubernamentales. Esta es la categoría que más se acerca si se compara con la realidad de la distribución demográfica del país porque cuenta con 62.5\% de participación en el año 2018 y la población panameña está compuesta por $68 \%$ de mestizos. 
De acuerdo al contexto de la publicidad, las marcas sitúan a los grupos étnicos principalmente en escenarios de vida cotidiana y trabajo. Otra gran parte de las personas aparecen sin fondo específico.

En cuestión de representatividad de género, las cifras generales son equitativas. La participación de los hombres en los grupos de los afrodescendientes, indígenas y mestizos es superior a la de las mujeres, mientras que, en los asiáticos y los blancos, domina el sexo femenino. Para el año 1998, el sexo masculino tenía mayor participación con una diferencia de $9.6 \%$ sobre el femenino. En la época actual, se invirtió de forma que las mujeres tienen un $6.4 \%$ de participación sobre los hombres.

Basada en la investigación realizada y los resultados obtenidos, sugerimos las siguientes recomendaciones:

-Los bancos de fotografías son una herramienta indispensable para la publicidad en la era actual. Se recomienda crear bancos de fotografías con imágenes que reflejen la población panameña y que integre la diversidad de modelos.

- Incentivar investigaciones relacionadas con la temática que nos permita observar y comparar la participación de grupos étnicos en la publicidad en otros medios y el impacto económico que podría generar.

-Incitar la realización de otros estudios similares en los que se analice el impacto del uso de estereotipos en consumidores y si influye en sus hábitos de compra. Indagar si el uso de otros grupos étnicos fuera de los suyos genera una impresión positiva o negativa hacia los productos pautados.

-Para el próximo Censo Nacional de Población que se realice, incluir datos estadísticos relacionados con los grupos mestizos, blancos y asiáticos. La información de estos tres grupos no estaba disponible en el censo anterior.

- Generar espacios de discusión sobre esta temática de manera sostenida.

\section{Agradecimientos}

Un inmenso agradecimiento a la Universidad Católica Santa María La Antigua, Vicerrectoría de Investigación y Postgrado por el apoyo incondicional para elaborar esta investigación. Al profesor asesor José Lasso y a los directores creativos entrevistado: Roberto Varela, Omar Polo, Luciano Carrizo, Julio Flores, Sussie Salado y Jan Valdés.

\section{Bibliografía}

Ley N4. Gaceta Oficial, 29 de enero de 1999

Bericat Alastuey, E. (1998). La integración de los métodos cuantitativo y cualitativo en la investigación social: significado y medida. España: Ariel. 
Cerrud, D. (2016). El 14.9\% de la población es afrodescendiente. Octubre 13, 2019, de La Estrella de Panamá Sitio web: http://laestrella.com.pa/panama/nacional/149-poblacion-

afrodescendiente/23939727

CIA. (2019). The World Factbook. Mayo 13, 2019, de CIA Sitio web:

https://www.cia.gov/library/publications/the-world-factbook/geos/pm.html

Codeluppi, V. (2007). El papel social de la publicidad1/The social role of advertising. Pensar la publicidad, 1(1), 149.

De León, A. (2016). VI Informe Clara González (2011-2013). Junio 19, 2019, de Instituto Nacional de la Mujer Sitio web: https://inamu.gob.pa/descargar-archivo/5754

Díez Aguado, J. (2015). La representación de la diversidad étnica en la publicidad comercial gráfica en España.

Erickson, B. F. (2001). La publicidad. Washington D. C.: Firmas Press.

García, D. S., \& Thomas, C. H. (2011). Psicología aplicada a la publicidad. Madrid: DYKINSON.

Hernández Sampieri, R, Fernández, C. \& Baptista, P. (2014). Metodología de la investigación sexta

edición. México: McGraw Hill.

INEC. (2010). Diagnóstico de la Población Indígena de Panamá con base en los Censos de

Población y Vivienda de 2010. Octubre 20, 2018, de Resultados Finales Básicos Sitio web: https://www.contraloria.gob.pa/INEC/Publicaciones/Publicaciones.aspx?ID_SUBCATEGORIA =59\&ID_PUBLICACION=360\&ID_IDIOMA=1\&ID_CATEGORIA=13

Jaramillo, V. S., Castillo, O. C., \& García, G. C. (2012). Análisis semiótico del discurso racista en la publicidad de Burberry Prorsum. Commons: revista de comunicación y ciudadanía digital, 1(1), 110140.

La Prensa. (2014). Panamá y sus diversas culturas. Octubre 17,2018, de La Prensa Sitio web: https://www.prensa.com/Panama-diversas-culturas_0_4048095182.html Ortega Carrillo, J. A., Victoria M., Juan S., \& Cristófol Rodríguez, C. (2011). Publicidad, educación y nuevas tecnologías. Madrid: Ministerio de Educación de España.

Pinilla, A.M. (2015). La pureza de nuestra diversidad. Octubre 20, 2018, de La Estrella de Panamá Sitio web: http://laestrella.com.pa/estilo/cultura/pureza-nuestra-diversidad/23866940

Treviño, R. (2010). Publicidad Comunicación integral en marketing tercera edición. México: McGraw Hill.

Van Dijk, T. (2001). Discurso y racismo. The Blackwell Companion to Racial and Ethnic Studies. Sitio web: http://www.discursos.org/Art/Discurso\%20y\%20racismo.pdf Wells, W., Moriarty S., \& Burnett J. (2007). Publicidad: principios y práctica séptima edición. México: Pearson Educación. 